\title{
Central, But Not Basolateral, Amygdala Is Critical for Control of Feeding by Aversive Learned Cues
}

\author{
Gorica D. Petrovich, ${ }^{1}$ Cali A. Ross, Pari Mody, ${ }^{2}$ Peter C. Holland, ${ }^{2}$ and Michela Gallagher ${ }^{2}$ \\ ${ }^{1}$ Department of Psychology, Boston College, Chestnut Hill, Massachusetts 02467-3807, and 2Department of Psychological and Brain Sciences, \\ Johns Hopkins University, Baltimore, Maryland 21218
}

\begin{abstract}
Environmental factors contribute to the motivation to eat and can override homeostatic signals to stimulate eating in sated states, or inhibit eating in states of hunger. In particular, stress, fear, and anxiety have been linked to suppression of eating and anorexia nervosa. Here, we use a rodent model of an aversive cue-induced cessation of feeding. In this setting, food-deprived rats suppress eating when presented with a tone [conditioned stimulus (CS)] that was previously paired with footshocks [unconditioned stimulus (US)]. To begin to delineate the underlying neural circuitry we examined the two regions of the amygdala with well known roles in associative learning-the central nucleus (CEA) and the basolateral area (BLA; includes the basolateral, basomedial, and lateral nuclei). We produced selective, bilateral, neurotoxic lesions of the CEA or BLA, and then trained these rats together with sham-lesioned controls in a behavioral protocol that allowed a test for food consumption in the presence of an aversive CS. Both sham- and BLA-lesioned rats showed inhibition of eating when presented with the CS. In contrast, bilateral, neurotoxic lesions of the CEA abolished this effect. These results demonstrate that the CEA, but not BLA, is critical for control of feeding by an aversive CS. Previously we demonstrated that enhancement of eating by an appetitive CS is dependent on the integrity of BLA, but not CEA. Those findings together with the current results show a double dissociation between amygdalar subsystems that control food consumption by appetitive and aversive learned cues.
\end{abstract}

\section{Introduction}

The motivation to eat is not only controlled by metabolic needs, but also by extrinsic factors that are not related to energy balance (Booth, 1989; Woods, 2005; Fehm et al., 2006; Berthoud, 2007). Environmental signals, including learned cues, can override homeostatic signals to stimulate eating in sated states, or inhibit eating in states of hunger (Weingarten, 1983; Stroebele and De Castro, 2004; Popkin et al., 2005; Petrovich and Gallagher, 2007). Persistent nonmetabolic modulation of neural systems that control feeding could be a contributing factor to dysregulation of appetite and eating disorders. Notably, stress, fear, and anxiety have been linked to both cessation of eating and anorexia nervosa (Job and Barnes, 1995; Hotta et al., 1999; Vallés et al., 2000; Klein and Walsh, 2004; Kaye, 2008), and enhancement of eating (Levine and Morley, 1981; Badiani et al., 1996; Polivy and Herman, 1999; Hagan et al., 2002; Dallman et al., 2005; Tamashiro et al., 2007). Here, we use a rodent model of decreased food consumption induced by learned aversive cues. In this setting, food-deprived rats curtail eating when presented with a discrete conditioned stimulus (CS) that has acquired aversive properties - a tone previously paired with footshocks [unconditioned stimulus (US)].

To define critical neural circuitry for such learned regulation of feeding, we focused on two regions of the amygdala with well

Received July 28, 2009; revised 0ct. 15, 2009; accepted 0ct. 22, 2009.

This research was supported by National Institute of Mental Health Grants MH67252 to G.D.P. and MH60179 to M.G. We thank Kathryn Sepelyak for contribution to behavioral analysis.

Correspondence should be addressed to Dr. Gorica D. Petrovich, Department of Psychology, Boston College, 140 Commonwealth Avenue, Chestnut Hill, MA 02467-3807. E-mail: gorica.petrovich@bc.edu.

DOI:10.1523/JNEUROSCI.3656-09.2009

Copyright $\odot 2009$ Society for Neuroscience ～0270-6474/09/2915205-08\$15.00/0 known roles in associative conditioning-the central nucleus (CEA) and the basolateral area (BLA; includes the basolateral, basomedial, and lateral nuclei) (Kapp et al., 1984; Davis, 1992; Killcross et al., 1997; Fendt and Fanselow, 1999; Holland and Gallagher, 1999; Gallagher, 2000; LeDoux, 2000; Davis and Whalen, 2001; Maren, 2001; Cardinal et al., 2002; Phillips et al., 2003; Balleine and Killcross, 2006). Recently, we demonstrated that only one of the two regions, the BLA, is critical for enhancement of eating driven by a learned appetitive cue (Holland et al., 2002). That finding is consistent with dissociable roles for CEA and BLA found in other behavioral preparations that rely on appetitive cues to modulate behavior (Holland and Gallagher, 1999; Gallagher, 2000; Parkinson et al., 2000; Cardinal et al., 2002; Balleine and Killcross, 2006). Because these regions together comprise a circuit critical for conditioned fear (Kapp et al., 1984; Davis, 1992; Fendt and Fanselow, 1999; LeDoux, 2000; Maren, 2001), we examined whether each of these regions is needed for cessation of eating under the influence of a learned aversive cue.

After sham or selective neurotoxic lesions of the CEA or BLA, rats received aversive training sessions in which either a tone or a light CS was paired with footshock. These sessions were interspersed among sessions in which the rats were given ad libitum access to food in distinctive appetitive test chambers, while food deprived. Finally, food consumption was evaluated in a test session that included presentations of the tone in the appetitive test chambers. Sham- and BLA-lesioned rats that had received toneshock pairings showed less food consumption than comparable rats that had received light-shock pairings, and the tone unpaired with shock. In contrast, all CEA-lesioned rats ate similar and 
substantial amounts of food, regardless of prior tone training. Thus, CEA, but not BLA, was critical for the inhibitory control of feeding by an aversive CS.

\section{Materials and Methods}

\section{Subjects}

Experimentally naive, male Long-Evans rats (Charles River Laboratories), weighing $\sim 300 \mathrm{~g}$ on arrival in the vivarium, were individually caged, maintained on a $12 \mathrm{~h}$ light/dark cycle, and given ad libitum access to food and water, except as otherwise noted. After 1 week of acclimation to the vivarium, rats were given bilateral, neurotoxic or sham lesions of the CEA or BLA and then allowed to recover for 2-3 weeks before undergoing behavioral procedures. Animal procedures were conducted in accordance with the National Institutes of Health Guidelines for the Care and Use of Laboratory Animals, and the guidelines of the Johns Hopkins University Institutional Animal Care and Use Committee.

\section{Surgical methods}

All surgeries were performed under aseptic conditions using isoflurane gas for induction and maintenance of anesthesia, with rats placed in a stereotaxic frame (Kopf Instruments). Lesions of the BLA were made with $12.5 \mathrm{mg} / \mathrm{ml} \mathrm{NMDA} \mathrm{(Sigma)} \mathrm{in} \mathrm{a} 0.1 \mathrm{M}$ PBS, and lesions of the CEA were made with $10 \mathrm{mg} / \mathrm{ml}$ Ibotenic Acid (Biosearch Technologies) in 0.1 M PBS. For the BLA, a total of $0.3 \mu \mathrm{l}$ was infused. The flat skull coordinates from bregma were as follows: anteroposterior (AP), $-2.70 \mathrm{~mm}$; mediolateral (ML), $\pm 4.80 \mathrm{~mm}$; and dorsoventral (DV; taken at the injection site), -8.70 and $-8.40 \mathrm{~mm} ; 0.2 \mu \mathrm{l}$ was infused at the deeper site and $0.1 \mu \mathrm{l}$ at the shallow site. For the CEA, a total of $0.2 \mu \mathrm{l}$ was infused. The flat skull coordinates from bregma were as follows: AP, $-2.00 \mathrm{~mm}$; $\mathrm{ML}, \pm 4.00 \mathrm{~mm}$; and DV (taken at the injection site), $-8.00 \mathrm{~mm}$. Sham lesions were made by infusing phosphate buffer vehicle alone into the CEA or the BLA. Infusions were made through a 30 gauge needle attached by a length of plastic tubing to a $10 \mu \mathrm{l}$ microsyringe (Hamilton) mounted on a syringe pump (Sage Instruments). After each infusion, the needle was left in place for $4 \mathrm{~min}$ to allow for diffusion. Immediately after surgery, rats were given buprenorphine (0.01-0.05 mg/kg, s.c.).

\section{Apparatus}

Appetitive and aversive sessions were conducted in different behavioral chambers that were located in different rooms. For the appetitive sessions a set of four identical behavioral chambers $(30 \times 24 \times 30 \mathrm{~cm}$; Coulbourn Instruments) was used. Each chamber had aluminum top and sides, a transparent Plexiglas back and front, and a dark navy Plexiglas panel placed on top of the grid floor so rats could not see or feel the grids. Each chamber contained a recessed food cup $(3.2 \times 4.2 \mathrm{~cm})$. The chambers were also equipped with a "house light" (4W light) mounted on the box wall $20 \mathrm{~cm}$ above the food cup. However, the house light was never illuminated during appetitive training sessions or during the test. Dim background illumination was provided by two $25 \mathrm{~W}$ red bulbs, each placed $1.5 \mathrm{~m}$ from the chambers. The chambers were wiped with $1 \%$ acetic acid (J.T. Baker) before an animal was introduced during each session. For the aversive training sessions we used four behavioral chambers $(30 \times 24 \times 30 \mathrm{~cm}$; Coulbourn Instruments) that were modified to differ from the chambers used in the appetitive sessions. These chambers had grid floors, black stripes on the Plexiglas front, and two black Plexiglas sheets were positioned to occlude the aluminum sides, and angled to create a tent-like enclosure. These chambers were equipped with a house light (4W light), which served as a signal for shocks for the rats in the Unpaired groups (see below, Behavioral training). No food cups were provided in the aversive training chambers, which were wiped with $5 \%$ ammonium hydroxide (v/v, from 28.0 to $30.0 \%$ stock, J.T. Baker) before each animal was placed inside for training. Dim background illumination was provided by two $25 \mathrm{~W}$ red bulbs, each placed $1.5 \mathrm{~m}$ from the chambers.

\section{Behavioral training}

A day before the behavioral protocol began, rats were given $\sim 1 \mathrm{~g}$ of the food pellets (formula PJPPP, $45 \mathrm{mg}$; Research Diets) in the home cage to familiarize them with those pellets. The protocol (Fig. 1) then consisted

\section{A Training}

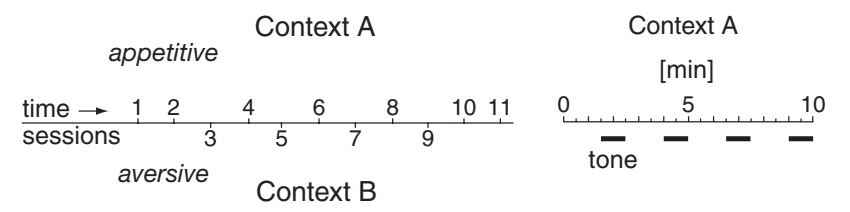

Figure 1. Experimental design. $\boldsymbol{A}$, Training. The behavioral protocol consisted of alternating appetitive and aversive sessions that were conducted in distinctive experimental chambers (contexts). During appetitive training sessions, food-deprived rats were given ad libitum access to food pellets. During aversive training, half of the rats from the three groups [BLA, CEA, and sham (SH) lesions] received electric footshocks signaled by a tone (Paired). The other half of the rats received footshocks that were not signaled by a tone (Unpaired), but instead a light preceded the shocks. Rats in both conditions received the same number of tone, shock, and light presentations during the overall training protocol. That protocol consisted of four sessions with habituation to the context occurring during the first session (S3). During the second session (S5), rats in the Paired (tone-paired) groups received house-light presentations, and rats in the Unpaired (tone-unpaired) groups received tone presentations to balance out exposure to all cues during the overall training protocol. The footshocks were administered during the third and fourth sessions $(S 7,59)$ when rats in Paired groups received tone-shock, while rats in the Unpaired groups received light-shock presentations. B, Food consumption test. Food-deprived rats were tested for food consumption during the 10 min test with 4 tone $C S$ presentations. The test was conducted in the appetitive context.

of 11 sessions with seven appetitive $(\mathrm{S} 1,2,4,6,8,10,11)$, and four aversive $(S 3,5,7,9)$ sessions presented in consecutive order. For each session, rats were transported from the vivarium to experimental rooms on a cart in groups of 4 . For the appetitive sessions rats were transported in their home cages, while for the aversive sessions they were transported in dark, plastic containers $(11.5 \times 7.5 \times 5$ inches $)$. Appetitive and aversive training sessions were conducted in distinct contexts; chambers differing in olfactory, visual, and tactile features were located in different rooms (see above).

Appetitive sessions. Before each session, rats were food deprived for $22 \mathrm{~h}$. Water was available ad libitum throughout the behavioral protocol in the home cage. For each session, rats were placed in the behavioral chamber with $7 \mathrm{~g}$ of food pellets in the food cup and allowed to consume food for $10 \mathrm{~min}$. After $10 \mathrm{~min}$, rats were taken out of the behavioral chamber, placed into the home cage, and transported back to the vivarium. Remaining food was removed from the food cup and weighed. Rats were allowed ad libitum access to food for at least $24 \mathrm{~h}$ between consecutive food-deprivations (S1, 2, and 10, 11).

Aversive sessions. During aversive training, half of the rats from the three groups (BLA, CEA, and sham lesions) received electric footshocks signaled by a tone (Paired). The other half of the rats received footshocks that were not signaled by a tone (Unpaired with respect to tone), but instead the shocks were preceded by a different nominal cue (house light). A signal for footshocks in the Unpaired groups was provided because we observed in pilot studies that rats receiving unsignaled footshocks in aversive sessions decreased consumption in subsequent appetitive sessions compared to rats that received signaled shocks.

The aversive training protocol consisted of four 10-min-long training sessions that were conducted on separate days. Rats were allowed ad libitum access to food and water for at least $24 \mathrm{~h}$ before each aversive session. In the first session (S3), rats were placed in the experimental chamber for $10 \mathrm{~min}$ to habituate them to the training context, which was different from the chambers used in the appetitive sessions (see above). To balance out exposure to all cues during training, in the second session (S5) rats in the Paired groups also received four random presentations of the light cue (house light, $60 \mathrm{~s}$ ), while rats in the Unpaired groups received four random presentations of the tone $(75 \mathrm{~dB} ; 60 \mathrm{~s})$. In each of the last two aversive sessions $(S 7,9)$, rats in the Paired groups received two tone presentations each immediately followed by an electric footshock $(0.8 \mathrm{~mA} ; 1 \mathrm{~s})$, while rats in the Unpaired groups received two presentations of the light cue each followed by a footshock. Thus, during aversive training all rats received equal number of tones, lights, and shocks but only rats in the Paired groups received tone-shock pairings. 

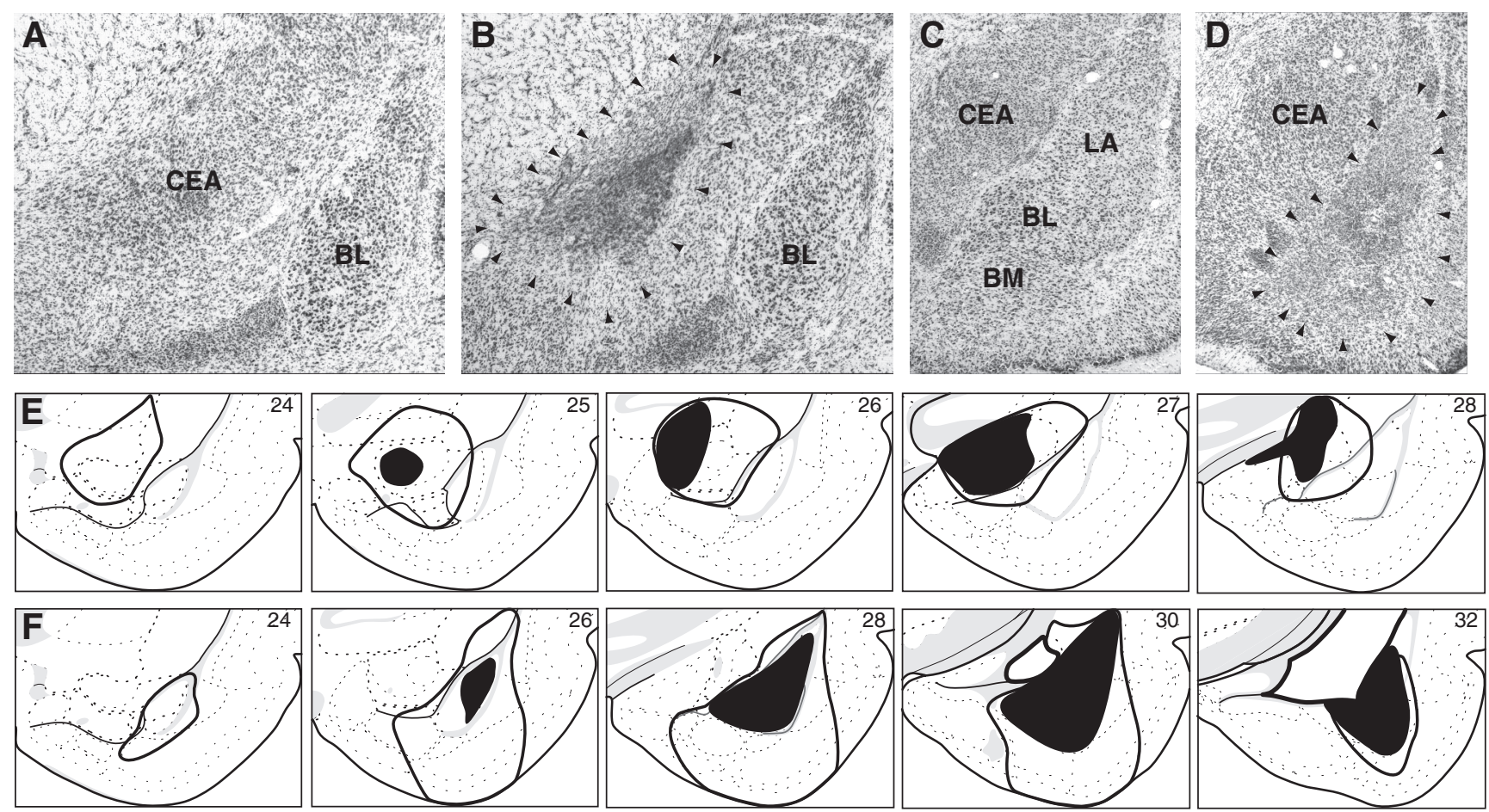

Figure 2. Histology. $\boldsymbol{A}-\boldsymbol{D}$, Representative photomicrographs of bilateral CEA $(\boldsymbol{B})$ and BLA $(\boldsymbol{D})$ lesions, and sham-lesioned controls $(\boldsymbol{A}, \boldsymbol{C}$. The arrowheads denote lesion borders. $\boldsymbol{B}$, The extent of the largest (enclosed black area) and smallest (filled black area) acceptable CEA (E) and BLA ( $\boldsymbol{F}$ ) lesions at several rostrocaudal levels. The plates were adapted from the atlas of $S$ wanson (2004), and the number in the upper right corner represents atlas level. BLA consists of BL, BM, and LA.

Food consumption test. Before consumption tests, rats were food deprived for $22 \mathrm{~h}$, while water remained available ad libitum. Rats were placed in the appetitive behavioral chamber with $7 \mathrm{~g}$ of food pellets in the food cup and allowed to consume food. During the test, four tones (75 $\mathrm{dB} ; 60 \mathrm{~s}$ ) were spaced at $90 \mathrm{~s}$ intervals. Behavior during the test was videotaped. After $10 \mathrm{~min}$, rats were taken out of the behavioral chamber, placed into the home cage and transported back to the vivarium. Remaining food was removed from the food cup and weighed.

The experiments were conducted in two replications with 48 rats in each. In one of the replications rats did not receive cue presentations during the second aversive session (S5; cue habituation) due to a technical malfunction. Otherwise, the replications were identical. One rat from the sham-lesioned, unpaired group had to be removed from the study because of incomplete data.

Behavioral analysis. Freezing and food-cup (nose-pokes into the food cup) behaviors were assessed for each rat during the test. Observations were made every $1.25 \mathrm{~s}$ during the entire $60 \mathrm{~s}$ of CSs, the $10 \mathrm{~s}$ immediately preceding the CSs (pre-CS periods), and an additional $25 \mathrm{~s}$ during the intertrial intervals (ITIs). At each observation, only one behavior was recorded. The observers were blind with respect to rat's lesion and pairing condition. The sum of all observations, which totals $6.33 \mathrm{~min}$ during the $10 \mathrm{~min}$ test represents "total time" in the text. The percentage of time the rats spent expressing each behavior was calculated for periods during CS, pre-CS, ITIs, or during total time.

Statistics. Behavioral data were analyzed using ANOVA, followed by Fisher's PLSD tests, where appropriate. In all cases, $p<0.05$ was considered significant.

Histological procedure. After completion of all behavioral procedures, rats were anesthetized and perfused intracardially with $0.9 \%$ saline, followed by $4 \%$ Formalin in $0.1 \mathrm{~m}$ PBS. The brains were removed and stored in the Formalin solution used for perfusion with $12 \%$ sucrose for $24 \mathrm{~h}$. The brains were sliced on a freezing microtome, and coronal sections $(30 \mu \mathrm{m})$ that were collected through the amygdala were mounted on slides and stained with thionin. Lesion placements were verified under a light microscope and drawn onto plates adapted from rat atlas (Swanson, 2004).

\section{Results}

\section{Histology}

Neurotoxic lesions were targeted to either the CEA or the BLA (includes the basolateral, basomedial, and lateral nuclei) of the amygdala. The extent of selective lesions of the CEA $(n=18)$ and BLA $(n=18)$ is shown in Figure 2. Lesions were rejected according to a preestablished criterion if the overall unilateral damage was $<50 \%$, or if that amount of minimal damage was only achieved in one hemisphere $(n=13)$. Nonselective lesions that produced substantial damage to both the CEA and BLA were also excluded from the study $(n=19)$. Of those, 7 rats in the paired and 6 in the unpaired condition had complete, or near complete BLA and CEA lesions, and are termed BLA+CEA in the text. No tissue damage was observed in sham-lesioned controls that received buffer injections into either the CEA or BLA.

\section{Behavioral training and tests}

After recovery from surgeries, rats were trained in the behavioral protocol (Fig. 1) with alternating appetitive and aversive sessions that were conducted in distinctive contexts (different behavioral chambers). The BLA-, CEA-, and sham-lesioned rats in the paired or unpaired groups had similar body weights at the beginning of training (Sham-P, $443 \pm 8 \mathrm{~g}$; Sham-U, $424 \pm 8$ g; BLA-P, $430 \pm 11$ g; BLA-U, $440 \pm 16$ g; CEA-P, $448 \pm 10$ g; CEA-U, $449 \pm 13 \mathrm{~g}$ ), and at the time of the consumption test at the end of the protocol (Sham-P, $455 \pm 8$ g; Sham-U, $437 \pm 9$ g; BLA-P, $437 \pm 12$ g; BLA-U, $460 \pm 17$ g; CEA-P, $465 \pm 11$ g; CEA-U, $470 \pm 17 \mathrm{~g})$. An ANOVA of body weight at the beginning of training with lesion type (BLA, CEA, or sham) and training condition (Paired or Unpaired) as factors revealed no effect of pairing, lesion, or lesion by pairing effect $(p>0.05$ for each). 


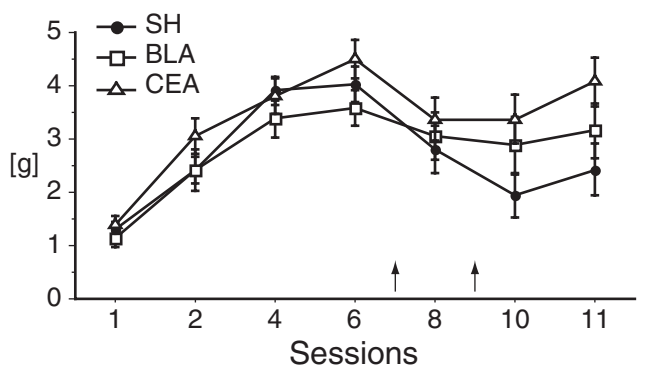

Figure 3. Food consumption (mean \pm SEM) during appetitive training sessions, which were conducted in Context A. Arrows point to the aversive sessions during which rats received electric foot-shocks in Context B. See Results for details. SH, Sham.

Table 1. Defecation during aversive training sessions

\begin{tabular}{lllllll}
\hline & SHP & SHU & BLAP & BLAU & CEA P & CEAU \\
\hline S3 & $1.3 \pm 0.4$ & $1.2 \pm 0.4$ & $0.8 \pm 0.5$ & $0.8 \pm 0.3$ & $0.6 \pm 0.3$ & $0.6 \pm 0.2$ \\
S5 & $0.6 \pm 0.3$ & $1.6 \pm 0.7$ & $1.5 \pm 0.5$ & $0.9 \pm 0.5$ & $0.6 \pm 0.5$ & $1.6 \pm 0.7$ \\
S7 & $7.1 \pm 0.6$ & $6.6 \pm 0.9$ & $5.3 \pm 1.2$ & $5.6 \pm 0.9$ & $3.8 \pm 1.4$ & $6.8 \pm 0.9$ \\
S9 & $8.0 \pm 1.0$ & $7.6 \pm 0.8$ & $6.3 \pm 1.3$ & $5.5 \pm 1.1$ & $5.9 \pm 1.2$ & $7.2 \pm 1.0$ \\
\hline
\end{tabular}

Rats in all groups excreted similar numbers of boli across training sessions, and rats in all groups excreted many more boli during sessions with electric footshocks $(\mathrm{S} 7, \mathrm{~S} 9)$ compared to sessions with no shocks $(\mathrm{S3}, \mathrm{S5})(p<0.02$ for all groups). See Results for details. SH, Sham.

Similarly, at the time of the consumption test there was no effect of pairing, lesion, or lesion by pairing effect ( $p>0.05$ for each).

During appetitive sessions food-deprived rats were given ad libitum access to food pellets. All groups of rats ate considerable amounts of food pellets during those sessions (Fig. 3). An ANOVA analysis of consumption with lesion condition as a factor revealed no statistically significant difference among the groups in any of the sessions ( $p>0.05$ for each session). Overall consumption was somewhat decreased in the sessions that followed aversive training with foot-shock exposure (indicated with arrows on Fig. 3). Comparison of consumption during the session that occurred before aversive training with shocks (S6) with the consumption during sessions that followed each aversive training with foot-shocks (S8, S10) showed that the decrease was statistically reliable for rats in all lesion conditions $(p<0.05$ for all lesions groups for S6 vs S8 and S6 vs S10).

During aversive training, Paired rats received tone-shock pairings and unshocked light presentations, and Unpaired rats received unshocked tone presentations and light-shock pairings. As a crude measure of responding to the shocks, we recorded defecation during these sessions, and found that all groups excreted similar numbers of boli, and more boli during sessions with shocks than during sessions without shocks (Table 1).

After aversive training, food-deprived rats were tested for food consumption in the appetitive context when four tone CS presentations occurred in the 10 min test (Fig. 4). Sham-lesioned rats that previously received tone-shock pairings (Paired group) inhibited consumption compared to rats in the sham control group that received tone and shocks unpaired during aversive training (Unpaired group). The BLA-lesions spared this CS suppression of feeding; rats in the BLA Paired group ate less than rats in the BLA Unpaired group. In contrast, all CEA-lesioned rats ate similar substantial amounts of food during the consumption tests (Fig. 4). An ANOVA of food consumption during the test with lesion type (BLA, CEA, or sham) and training condition (Paired or Unpaired) as factors revealed a main effect of pairing $\left(F_{(1,57)}=\right.$ 11.747, $p<0.002)$, but no lesion $\left(F_{(2,57)}=2.381, p=0.1016\right)$ or lesion by pairing effect $\left(F_{(2,57)}=1.381, p=0.2596\right)$. Subsequent

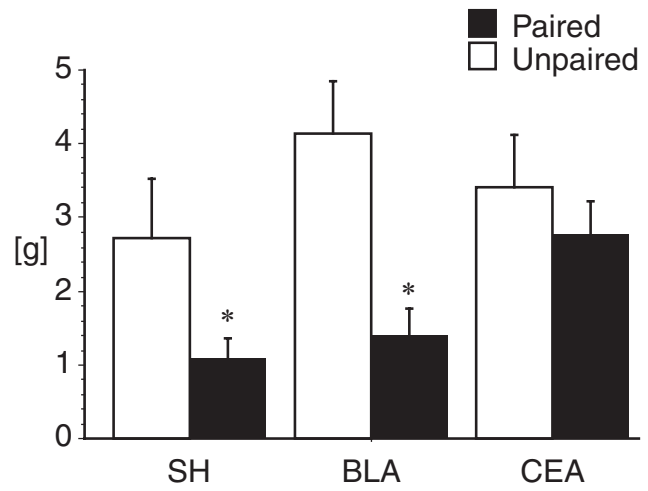

Figure 4. Food consumption (mean \pm SEM) during test with CS presentations in Context $A$. An asterisk indicates significant difference in consumption between rats in paired and unpaired groups ( $p<0.05$ ). See Results for details. SH, Sham.

comparisons revealed that sham-lesioned rats in the paired group consumed significantly less food than sham-lesioned rats in the unpaired group $(p<0.05)$. Similarly, the consumption by BLAlesioned rats in the paired group was significantly less than the consumption by BLA-lesioned rats in the unpaired group $(p<$ 0.01 ), while there was no difference in consumption by rats with CEA lesions in Paired and Unpaired groups $(p>0.05)$. Furthermore, food consumption did not differ for sham and BLA lesion groups in the Paired condition $(p>0.05)$ while the CEAlesioned rats in the Paired group ate significantly more than sham-lesioned Paired rats $(p<0.05)$.

We also measured laboratory chow consumption in the home cage during the first hour immediately after the tests. Rats in all groups consumed similar amounts of laboratory chow in the home cages after tests (Sham-P, $5.6 \pm 0.4 \mathrm{~g}$; Sham-U, $5.8 \pm 1.0 \mathrm{~g}$; BLA-P, $6.7 \pm 1.0 \mathrm{~g}$; BLA-U, $5.7 \pm 0.5$ g; CEA-P, $6.6 \pm 0.5$ g; CEA-U, $5.8 \pm 1 \mathrm{~g})$. An ANOVA analysis of laboratory chow consumption post-test with lesion type (BLA, CEA, or sham) and training condition (Paired or Unpaired) as factors revealed no effect of lesion, paring, or lesion by paring interaction $(p>0.05$ for each).

Consistent with the above findings, we observed that rats whose lesions extended across both the BLA and CEA (BLA+ CEA) showed impairment in inhibition of eating under the aversive tone, similar to the CEA lesions. All rats that had combined BLA and CEA lesions ate substantial amounts of food during the tests (BLA+CEA Paired, $3.8 \pm 1.0$ g; BLA + CEA Unpaired, $4.3 \pm$ 0.7 g) (see Fig. 6).

In addition to food consumption, during the tests we measured conditioned freezing behavior, a species-typical defense response characterized by the absence of all movement except that required for breathing (Fanselow, 1984). Rats across all lesion and pairing conditions showed minimal freezing during the pre-CS period before the first CS presentation $(<3 \%)$. As a reflection of conditioned fear, sham-lesioned rats in the paired group spent a substantial amount of time freezing during the CS presentations compared to rats in the unpaired sham group (Fig. $5 A$; see also supplemental Fig. $1 A$ for behavior during individual CSs, available at www.jneurosci.org as supplemental material). Consistent with much prior research (see Discussion), lesions of the BLA, or of the CEA, abolished this effect; all rats with those lesions showed minimal freezing during the CS, comparable to that of unpaired sham-lesioned rats (Fig. 5A). An ANOVA of the percentage of time spent freezing during the CS presentation with lesion type (BLA, CEA, or sham) and training condition (Paired 


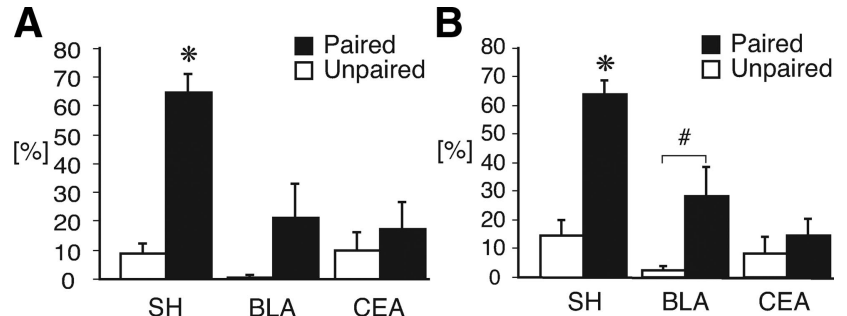

Figure 5. Conditioned freezing behavior (mean \pm SEM) during food consumption test. $A$, Graph shows percentage of the time spent freezing during the first CS presentation. $B$, Graph shows percentage of the total time spent freezing during the test. Observations were made during $\sim 6.3 \mathrm{~min}$ of the $10 \mathrm{~min}$ test (during $\mathrm{CSs}$, pre-CSs, and portions of the ITIs, see Materials and Methods for details), and the total percentage of the time spent freezing during that period is shown. An asterisk indicates significant difference in the amount of freezing between rats in the SH-paired and all other groups $(p<0.0001)$, and the pound symbol indicates difference between paired and unpaired rats with BLA lesions $(p<0.05)$. See Results for details. SH, Sham.

or Unpaired) as factors revealed main effects of lesion $\left(F_{(2,57)}=\right.$ 9.786, $p=0.0002)$, pairing $\left(F_{(1,57)}=24.629, p<0.0001\right)$, and a lesion by pairing interaction $\left(F_{(2,57)}=7.289, p=0.0015\right)$. Subsequent comparisons showed greater freezing by sham-lesioned rats in the paired group relative to all other groups $(p<0.0001)$. The difference in the amount of freezing between other groups was not statistically significant $(p>0.05)$.

Analysis of the total amount of time spent freezing during the test yielded similar results (Fig. $5 B$ ). Behavioral observations were made during $6.3 \mathrm{~min}$ of the $10 \mathrm{~min}$ test (during CSs, pre-CSs, and portions of the ITI intervals, see Materials and Methods for details), and the total percentage of time spend freezing during that period is shown. An ANOVA of the percentage of total time spent freezing during the test with lesion type (BLA, CEA, or sham) and training condition (Paired or Unpaired) as factors revealed significant effect of lesion $\left(F_{(2,57)}=14.233, p<0.0001\right)$, pairing $\left(F_{(1,57)}=31.111, p<0.0001\right)$, and lesion by pairing interaction $\left(F_{(2,57)}=6.926, p=0.002\right)$. Subsequent comparisons confirmed that the sham-lesioned rats in the paired group showed significantly greater freezing compared to all other groups $(p<$ 0.0001), while the BLA-lesioned rats in the unpaired group rats appeared to freeze less than sham or CEA rats in unpaired groups, that effect was not statistically reliable for freezing during CS, or total freezing during test $(p>0.05$ for BLA-U vs Sham-U, and BLA-U vs CEA-U). However, the difference between BLAlesioned rats in the unpaired group and BLA-lesioned rats in the paired condition was statistically significant $(p<0.05)$. While an interpretation of that observation is not certain, it might reflect a BLA role in processing of contextual information necessary for discrimination between the different environments in which tests and aversive training were conducted.

As expected, rats with combined lesions of the BLA and CEA (BLA + CEA) in paired and unpaired conditions showed minimal freezing behavior during the CS presentation (BLA + CEA Paired, $3.9 \pm 4 \%$; BLA + CEA Unpaired, $1 \pm 1 \%$ ) and during the $10 \mathrm{~min}$ test $(\mathrm{BLA}+\mathrm{CEA}$ Paired, $3.7 \pm 2 \%$; BLA +CEA Unpaired, $0.7 \pm 0.7 \%$ ) (Fig. 6; see supplemental Fig. $1 A$ for behavior during individual CSs, available at www. jneurosci.org as supplemental material).

The current data show that BLA lesions produced divergent effects on freezing and eating in the presence of an aversive CS (tone)-those lesions greatly decreased CS-induced freezing compared to the shams, but left CS-inhibition of eating intact and comparable to the shams. In contrast, the CEA lesions abol-
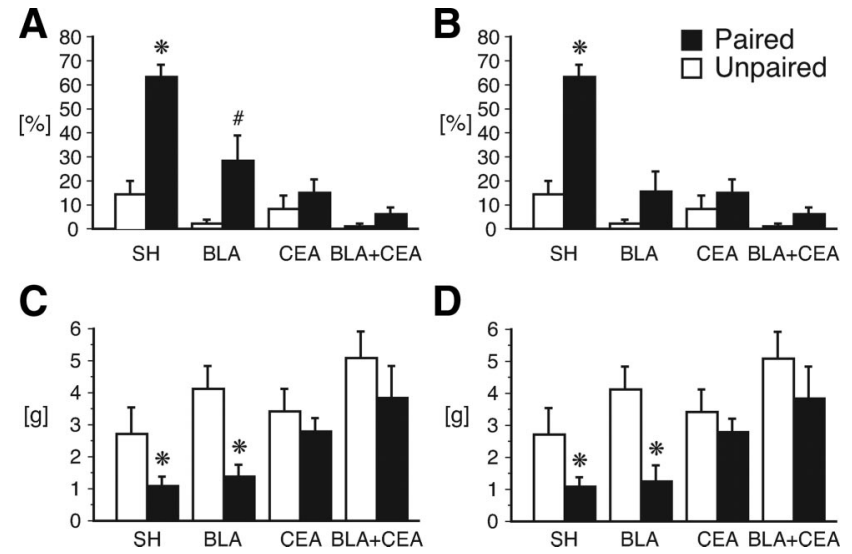

Figure 6. $\quad \boldsymbol{A}-\boldsymbol{D}$, Freezing behavior $(\boldsymbol{A}, \boldsymbol{B})$ and food consumption $(\boldsymbol{C}, \boldsymbol{D})$ during test with $C$ presentations. The graphs show comparison of behavior for the complete set of $B L A$ rats $(A, C)$ with behavior of subset of $B L A$ rats $(\boldsymbol{B}, \boldsymbol{D})$ that was created to match the average freezing of rats with BLA lesions to the CEA-P and Sham-U groups (two BLA rats with the highest freezing scores removed). $\boldsymbol{A}, \boldsymbol{B}$, Graphs show percentage of the total time spent freezing during the test. $0 \mathrm{~b}$ servations were made during $\sim 6.3 \mathrm{~min}$ of the 10 min test (see Materials and Methods for details), and the total percentage of time spent freezing during that period is shown. An asterisk indicates significant difference in the amount of freezing between rats in the sham-paired and all other groups ( $p<0.0001$ ), and the pound symbol indicates difference between BLA lesioned rats in paired and rats in unpaired BLA, unpaired CEA, and the BLA+CEA rats ( $p<$ 0.05). The BLA-lesioned rats in paired group were not different from sham unpaired, or CEA paired ( $p>0.05$ ). C, D, Food consumption during test with CS presentations in Context A. An asterisk indicates significant difference in consumption between rats in paired and unpaired groups $(p<0.05)$. SH, Sham.

ished both behavioral responses to the CS. However, the BLAlesioned rats in the paired condition exhibited slightly more freezing than the CEA-lesioned paired group $(p>0.05)$, or sham-lesioned unpaired controls $(p>0.05)$ during the test. Thus, to examine whether the residual freezing in the BLAlesioned rats might contribute to inhibition of eating, we removed two rats with the highest freezing scores to match freezing exhibited by the remaining rats $(n=6)$ with that of the CEAlesioned paired group and sham-lesioned unpaired group (Fig. $6 B$ ), and then examined whether any change in eating scores was observed as a consequence (Fig. 6D). The average pellet consumption of the remaining BLA rats was almost identical (even slightly lower) compared to the average consumption exhibited by the entire BLA group (Fig. $6 C, D$ ). Thus, those data indicate that inhibition of eating in rats with BLA lesions was not due to residual freezing in that treatment condition.

Finally, we also analyzed time spent in food-cup (food-cup behavior) during the test (Fig. 7; see supplemental Fig. $1 B$ for behavior during individual CSs, available at www.jneurosci.org as supplemental material). We found that the overall pattern of food-cup behavior was similar to the eating behavior observed during the test such that the behavior of the BLA-lesioned rats was similar to that of sham-lesioned controls, while behavior of the CEA-lesioned rats was similar to the BLA+CEA-lesioned rats (Fig. 7A). An ANOVA of total food-cup behavior during the test with lesion type (BLA, CEA, sham, or BLA+CEA) and training condition (Paired or Unpaired) as factors revealed a main effect of pairing $\left(F_{(1,68)}=14.519, p=0.0003\right)$ and lesion $\left(F_{(3,68)}=\right.$ 3.303, $p=0.0254)$, but no lesion by pairing effect interaction $\left(F_{(3,68)}=0.135, p=0.9389\right)$. Subsequent comparisons revealed that sham and BLA rats in tone-paired condition inhibited foodcup behavior compared to the rats in Unpaired groups $(p<0.05$ for Sham-P vs Sham-U and BLA-P vs BLA-U), while a slight 
A
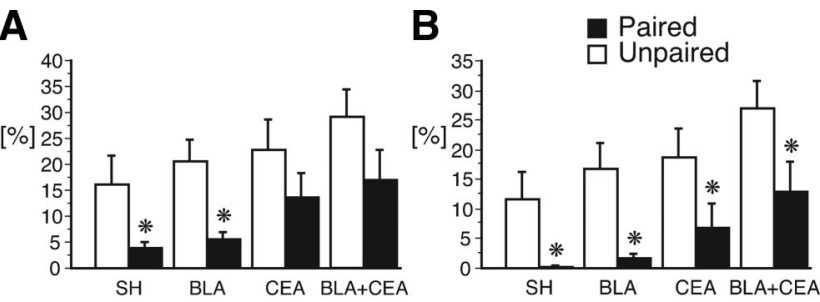

C

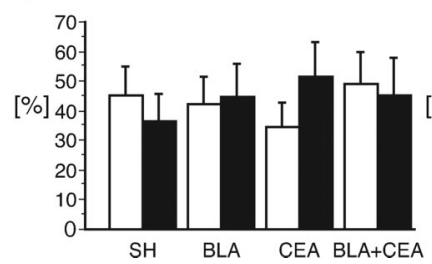

\section{D}

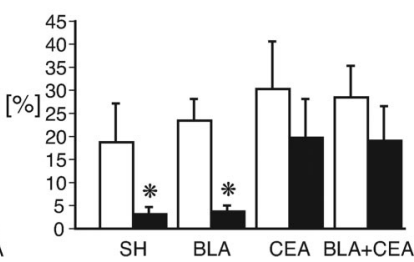

Figure 7. Food-cup behavior during test with CS presentations. A, Graph shows percentage of the total time spent expressing food-cup behavior during the test. Observations were made during $\sim 6.3$ min of the 10 min test (see Materials and Methods for details), and percentage of the time spent expressing food-cup during that period is shown. $\boldsymbol{B}$, Graph shows percentage of the time spent expressing food-cup behavior during the CSs. C, Graph shows percentage of the time spent expressing food-cup behavior before first CS presentation. $D$, Graph shows percentage of the time spent expressing food-cup behavior during intertrial intervals after $C S$ presentations. An asterisk indicates significant difference between rats in paired and unpaired groups $(p<0.05)$. SH, Sham.

decrease in Paired CEA- and BLA+CEA-lesioned rats was not statistically different from the Unpaired $(p>0.05)$.

Detailed analysis revealed that all groups showed similar foodcup behavior before the first CS presentation (Fig. 7C). All groups that had received shock previously paired with tone decreased food-cup behavior during CSs (Fig. 7B, see also supplemental Fig. $1 B$ for behavior during individual CSs, available at www. jneurosci.org as supplemental material). However, after the first CS presentation, Sham-P and BLA-P rats continued to suppress food-cup behavior during the intertrial periods that followed CSs, while the CEA and BLA+CEA did not (Fig. 7D). Results from the statistical analysis support these observations. An ANOVA of food-cup behavior before that first CS presentation with lesion type (BLA, CEA, sham, or BLA+CEA) and training condition (Paired or Unpaired) as factors found no effect of pairing $\left(F_{(1,68)}=0.053, p=0.8184\right)$, lesion $\left(F_{(3,68)}=0.121, p=\right.$ $0.362)$, or lesion by pairing effect $\left(F_{(3,68)}=0.602, p=0.6161\right)$. An ANOVA of food-cup behavior during the intertrial periods after the first CS presentation with lesion type (BLA, CEA, sham, or BLA + CEA) and training condition (Paired or Unpaired) as factors revealed a main effect of pairing $\left(F_{(1,68)}=8.291, p=0.0053\right)$, but no significant lesion $\left(F_{(3,68)}=2.388, p=0.0765\right)$ or lesion by pairing interaction $\left(F_{(3,68)}=0.232, p=0.697\right)$. Subsequent comparisons revealed that BLA and shams inhibited food-cup behavior compared to the Unpaired controls ( $p<0.05$ for Sham-P vs Sham-U and BLA-P vs BLA-U), while the CEA and BLA+CEA did not $(p>0.05$ for CEA-P vs CEA-U and BLA+CEA-P vs $\mathrm{BLA}+\mathrm{CEA}-\mathrm{U})$.

An ANOVA of food-cup behavior during the CSs with lesion type (BLA, CEA, sham, or BLA+CEA) and training condition (Paired or Unpaired) as factors revealed a main effect of pairing $\left(F_{(1,68)}=22.918, p<0.0001\right)$, and lesion $\left(F_{(3,68)}=4.573, p=\right.$ $0.0056)$, but no lesion by pairing interaction $\left(F_{(3,68)}=0.125, p=\right.$ 0.9452). Furthermore, all rats in Paired groups spent significantly less time expressing food-cup behavior compared to Unpaired controls $(p<0.05)$.
These data provide further support for different roles of the BLA and CEA in the control of behavior by aversive CSs.

\section{Discussion}

Here, we present evidence that the control of eating by an aversive cue critically depends on the CEA, but still occurs when the BLA alone is damaged. We used a preparation in which food-deprived rats inhibit food consumption when presented with a tone (CS) previously paired with footshocks (US). Bilateral, neurotoxic, lesions of the CEA abolished this effect. In contrast, rats with bilateral, neurotoxic lesions of the BLA showed CS-driven inhibition of food consumption. The cessation of eating in this preparation is not merely a consequence of immobilization due to CSinduced conditioned freezing. Both the BLA- and CEA-lesioned rats greatly reduced freezing compared to the sham-lesioned rats, but only the CEA-lesioned rats failed to control feeding in the presence of an aversive CS. These data suggest that the influence of CS on eating is independent of CS-induced freezing, and engages dissociable amygdalar subsystems.

Recently, we reported a dissociation between the involvement of the BLA and CEA in another setting in which learned cues control eating. In contrast to the current findings, enhancement of eating by an appetitive CS is dependent on the integrity of the BLA, but not CEA (Holland et al., 2002; Holland and Petrovich, 2005). Thus, that finding together with the current data demonstrate a double dissociation between amygdalar subsystems that control food consumption by appetitive and aversive CSs. In both paradigms, the CS modulates feeding based on motivational properties acquired through Pavlovian conditioning. However, the CS effects on feeding in the appetitive and aversive conditioning protocols are opposite, and are most likely mediated via distinct motivational processes. In that regard, cessation of eating in anticipation of danger is an adaptive response that is part of a broad recruitment of preparatory and motivational systems critical for defensive behavior. In contrast, CS-driven eating, which depends on BLA, has specificity for the food US used in the training protocol rather than a more general process affecting consumption of all food (Petrovich et al., 2007). Thus, these processes might relate to the recently proposed roles for the CEA and BLA in general and specific motivation, respectively (Balleine and Killcross, 2006; Seymour and Dolan, 2008).

In contrast to dissociable roles in the control of eating, both the BLA and CEA are necessary for conditioned freezing. Here we confirmed that lesions of either the CEA or BLA abolished CS driven freezing behavior in agreement with previous work (Maren, 2001; Fanselow and Poulos, 2005; Lee et al., 2005). These results, however, do not distinguish whether the two structures act independently or whether the communication between the CEA and BLA is critical; impairments produced by elimination of either structure could be due to disruption of BLA-to-CEA connections as suggested in some circuit models of conditioned fear (LeDoux, 2000; Maren, 2001; Fanselow and Poulos, 2005) or could be due to disruption of separate, parallel BLA- and CEAcircuits (Killcross et al., 1997; Phillips et al., 2003; Balleine and Killcross, 2006; Seymour and Dolan, 2008) whose simultaneous activation might be necessary for conditioned freezing. A functional disconnection of the BLA-CEA pathway, which would resolve this issue, to our best knowledge, has not been performed.

Our data, nonetheless, indicate that the critical circuitry for the ability of an aversive CS to modulate feeding includes CEA independent of the BLA. In that regard, it is notable that our BLA lesions included damage to the basomedial (BM), basolateral (BL), and lateral (LA) nuclei. Importantly, the LA does not send 
direct input to the main CEA output, the medial part of the CEA (Pitkänen et al., 1995; Swanson and Petrovich, 1998), but reaches it mainly via relays in the BL and BM. Thus, our BLA lesions disrupted the LA-BM/BN-CEA pathways, making it unlikely that these connections contribute to the CEA role observed here. Additionally, there is evidence that the CEA itself can support aversive learning induced by tone-shock pairings. It receives and processes sensory information independent of the BLA (Swanson and Petrovich, 1998; Rosenkranz et al., 2006), and its role in aversive cue acquisition was recently demonstrated in a conditioned fear paradigm (Wilensky et al., 2006). Consequently, multiple CS-US associations might be generated concurrently within different amygdalar subsystems to support somewhat distinct CS functions.

The CEA in turn could influence feeding mechanisms via multiple routes. Its output network includes direct projections to the brainstem, lateral hypothalamus, and bed nuclei of the stria terminalis (Swanson and Petrovich, 1998; Dong et al., 2001), as well as indirect pathways to the paraventricular nucleus of the hypothalamus (Prewitt and Herman, 1998; Dong et al., 2001). Other functional evidence shows the CEA has access to regulation of a conditioned insulin response (Roozendaal et al., 1990) and can modulate $\mu$-opioid-induced feeding in the nucleus of the solitary tract (Giraudo et al., 1998b) and in the paraventricular hypothalamic nucleus (Giraudo et al., 1998a). Furthermore, a neuronal network that supports dehydration-induced anorexia also includes the CEA (Watts, 2001; Watts et al., 2007).

It is instructive to compare potential circuits for feeding regulation with those for other functions of the CEA in defensive conditioning. In another study (Petrovich et al., 2006) (our unpublished observations) we observed CS-produced inhibition of eating in the absence of conditioned freezing after damage to a midbrain target of CEA known to be critical to conditioned freezing, the ventrolateral region of the periaqueductal gray (PAGvl) (Amorapanth et al., 1999). Bilateral electrolytic lesions of the PAGvl, made after training in which rats received tone-footshock pairings, abolished conditioned freezing during a test with tone (CS) presentations. Nevertheless, both sham- and PAGvl-lesioned rats inhibited eating in the presence of the CS (Petrovich et al., 2006). Thus, additional studies are needed to define the outputs of CEA that modulate feeding in this paradigm.

Here, we used a preparation that results in inhibition of eating in response to a stress cue. However, the relationship between stress and eating is complex, and could result in enhancement of eating, and even obesity in chronic settings (Levine and Morley, 1981; Badiani et al., 1996; Polivy and Herman, 1999; Hagan et al., 2002; Dallman et al., 2005; Tamashiro et al., 2007). Interestingly, results from human studies suggest that dieting critically modulates stress response. Individuals defined as restrained eaters (chronic dieters) show enhancement of eating in response to stress that typically inhibits eating in control (nonrestrained) eaters (for review, see Polivy and Herman, 1999).

Our findings highlight an amygdalar subsystem critical for short-term anorexia triggered by an impending aversive event. The CEA and its associated network may also be critical for longer-term suppression of eating, triggered by repeated stress, fear, and anxiety. As such, dysfunction within this system could contribute to anorexia nervosa, which is associated with affective disorders and depression (Klein and Walsh, 2004; Kaye, 2008). Indeed, recent findings from human brain imaging studies support an amygdalar role in anorexia nervosa. Abnormal amygdalar functioning (Takano et al., 2001) and a decrease in its volume
(Giordano et al., 2001) in anorexia nervosa patients have been reported. Others have reported greater amygdala activation among anorexia patients in a symptom provocation protocol based on a core feature of the disorder involving fear of becoming fat despite being underweight (Seeger et al., 2002). Interestingly, in another study the amygdala was recruited in non-eating disordered young women when viewing pictures of slim/idealized female bodies, and its activity was correlated with increased anxiety induced by those images (Friederich et al., 2007).

In conclusion, here we provide evidence that a specific region of the amygdala, the CEA, but not the BLA, is critical for the ability of a learned cue to induce short-term cessation of feeding in food-deprived rodents. The animal model may also be relevant to regulation of eating in humans, and provides a framework for defining the critical brain substrates that could lead to conditions affecting food intake in other settings, such as anorexia.

\section{References}

Amorapanth P, Nader K, LeDoux JE (1999) Lesions of periaqueductal gray dissociate-conditioned freezing from conditioned suppression behavior in rats. Learn Mem 6:491-499.

Badiani A, Jakob A, Rodaros D, Stewart J (1996) Sensitization of stressinduced feeding in rats repeatedly exposed to brief restrain: the role of corticosterone. Brain Res 710:35-44.

Balleine BW, Killcross S (2006) Parallel incentive processing: an integrated view of amygdala function. Trends Neurosci 29:272-279.

Berthoud HR (2007) Interactions between the "cognitive" and "metabolic" brain in the control of food intake. Physiol Behav 91:486-498.

Booth DA (1989) Mood- and nutrient-conditioned appetities. Cultural and physiological bases for eating disorders. Ann NY Acad Sci 575:122-135.

Cardinal RN, Parkinson JA, Hall J, Everitt BJ (2002) Emotion and motivation: the role of the amygdala, ventral striatum, and prefrontal cortex. Neurosci Biobehav Rev 26:321-352.

Dallman MF, Pecoraro NC, la Fleur SE (2005) Chronic stress and comfort foods: self-medication and abdominal obesity. Brain Behav Immun 19:275-280.

Davis M (1992) The role of the amygdala in fear and anxiety. Annu Rev Neurosci 15:353-375.

Davis M, Whalen PJ (2001) The amygdala: vigilance and emotion. Mol Psychiatry 6:13-34.

Dong HW, Petrovich GD, Swanson LW (2001) Topography of projections from amygdala to bed nuclei of the stria terminalis. Brain Res Rev 38:192-246.

Fanselow MS (1984) What is conditioned fear? Trends Neurosci 7:400-402.

Fanselow MS, Poulos AM (2005) The neuroscience of mammalian associative learning. Annu Rev Psychol 56:207-234.

Fehm HL, Kern W, Peters A (2006) The selfish brain: competition for energy resources. Prog Brain Res 153:129-140.

Fendt M, Fanselow MS (1999) The neuroanatomical and neurochemical basis of conditioned fear. Neurosci Biobehav Rev 23:743-760.

Friederich HC, Uher R, Brooks S, Giampietro V, Brammer M, Williams SC, Herzog W, Treasure J, Campbell IC (2007) I'm not as slim as that girl: neural bases of body shape self-comparison to media images. Neuroimage 37:674-681.

Gallagher M (2000) The amygdala and associative learning. In: The amygdala: a functional analysis (Aggleton JP, ed), pp 311-329. New York: Oxford UP.

Giordano GD, Renzetti P, Parodi RC, Foppiani L, Zandrino F, Giordano G, Sardanelli F (2001) Volume measurement with magnetic resonance imaging of hippocampus-amygdala formation in patients with anorexia nervosa. J Endocrinol Invest 24:510-514.

Giraudo SQ, Billington CJ, Levine AS (1998a) Effects of the opioid antagonist naltrexone on feeding induced by DAMGO in the central nucleus of the amygdala and in the paraventricular nucleus in the rat. Brain Res 782:18-23.

Giraudo SQ, Kotz CM, Billington CJ, Levine AS (1998b) Association between the amygdala and nucleus of the solitary tract in $\mu$-opioid induced feeding in the rat. Brain Res 802:184-188.

Hagan MM, Wauford PK, Chandler PC, Jarrett LA, Rybak RJ, Blackburn K 
(2002) A new animal model of binge eating: key synergistic role of past caloric restriction and stress. Physiol Behav 77:45-54.

Holland PC, Gallagher M (1999) Amygdala circuitry in attentional and representational processes. Trends Cogn Sci 3:65-73.

Holland PC, Petrovich GD (2005) A neural systems analysis of the potentiation of feeding by conditioned stimuli. Physiol Behav 86:747-761.

Holland PC, Petrovich GD, Gallagher M (2002) The effects of amygdala lesions on conditioned stimulus-potentiated eating in rats. Physiol Behav 76:117-129.

Hotta M, Shibasaki T, Arai K, Demura H (1999) Corticotropin-releasing factor receptor type 1 mediates emotional stress-induced inhibition of food intake and behavioral changes in rats. Brain Res 823:221-225.

Job RF, Barnes BW (1995) Stress and consumption: inescapable shock, neophobia, and quinine finickiness in rats. Behav Neurosci 109:106-116.

Kapp BS, Pascoe JP, Bixler MA (1984) The amygdala: a neuroanatomical systems approach to its contribution to aversive conditioning. In: The neuropsychology of memory (Squire L, Butters N, eds), pp 473-488. New York: The Guilford.

Kaye W (2008) Neurobiology of anorexia and bulimia nervosa. Physiol Behav 94:121-135.

Killcross S, Robbins TW, Everitt BJ (1997) Different types of fearconditioned behaviour mediated by separate nuclei within amygdala. Nature 388:377-380.

Klein DA, Walsh BT (2004) Eating disorders: clinical features and pathophysiology. Physiol Behav 81:359-374.

LeDoux JE (2000) Emotion circuits in the brain. Annu Rev Neurosci 23:155-184.

Lee JL, Dickinson A, Everitt BJ (2005) Conditioned supression and freezing as measures of aversive Pavlovian conditioning: effects of discrete amygdala lesions and overtraining. Behav Brain Res 159:221-233.

Levine AS, Morley JE (1981) Stress-induced eating in rats. Am J Physiol 241:R72-R76.

Maren S (2001) Neurobiology of Pavlovian fear conditioning. Annu Rev Neurosci 24:897-931.

Parkinson JA, Robbins TW, Everitt BJ (2000) Dissociable roles of the central and basolateral amygdala in appetitive emotional learning. Eur J Neurosci 12:405-413.

Petrovich GD, Gallagher M (2007) Control of food consumption by learned cues: a forebrain-hypothalamic network. Physiol Behav 91:397-403.

Petrovich GD, Ross CA, Holland PC, Gallagher M (2006) Central but not basolateral amygdala is critical for control of feeding by aversive, conditioned cues. Soc Neurosci Abstr 32:373.7.

Petrovich GD, Ross CA, Gallagher M, Holland PC (2007) Learned contextual cue potentiates eating in rats. Physiol Behav 90:362-367.

Phillips AG, Ahn S, Howland JG (2003) Amygdalar control of the mesocorticolimbic dopamine system: parallel pathways to motivated behavior. Neurosci Biobehav Rev 27:543-554.

Pitkänen A, Stefanacci L, Farb CR, Go GG, LeDoux JE, Amaral DG (1995) Intrinsic connections of the rat amygdaloid complex: projections originating in the lateral nucleus. J Comp Neurol 356:288-310.
Polivy J, Herman CP (1999) Distress and eating: why do dieters overeat? Int J Eat Disord 26:153-164.

Popkin BM, Duffey K, Gordon-Larsen P (2005) Environmental influences on food choice, physical activity and energy balance. Physiol Behav 86:603-613.

Prewitt CM, Herman JP (1998) Anatomical interactions between the central amygdaloid nucleus and the hypothalamic paraventricular nucleus of the rat: a dual tract-tracing analysis. J Chem Neuroanat 15:173-185.

Roozendaal B, Oldenburger WP, Strubbe JH, Koolhaas JM, Bohus B (1990) The central amygdala is involved in the conditioned but not in the mealinduced cephalic insulin response in the rat. Neurosci Lett 116:210-215.

Rosenkranz JA, Buffalari DM, Grace AA (2006) Opposing influence of basolateral amygdala and footshock stimulation on neurons of the central amygdala. Biol Psychiatry 59:801-811.

Seeger G, Braus DF, Ruf M, Goldberger U, Schmidt MH (2002) Body image distortion reveals amygdala activation in patients with anorexia nervosa-a functional magnetic resonance imaging study. Neurosci Lett 326:25-28.

Seymour B, Dolan R (2008) Emotion, decision making, and the amygdala. Neuron 58:662-671.

Stroebele N, De Castro JM (2004) Effect of ambience on food intake and food choice. Nutrition 20:821-838.

Swanson LW (2004) Brain maps: structure of the rat brain. a laboratory guide with printed and electronic templates for data, models and schematics, Ed 3 with CD-ROM. Amsterdam: Elsevier.

Swanson LW, Petrovich GD (1998) What is the amygdala? Trends Neurosci 21:323-331.

Takano A, Shiga T, Kitagawa N, Koyama T, Katoh C, Tsukamoto E, Tamaki N (2001) Abnormal neuronal network in anorexia nervosa studied with I-123-IMP SPECT. Psychiatry Res 107:45-50.

Tamashiro KL, Hegeman MA, Nguyen MM, Melhorn SJ, Ma LY, Woods SC, Sakai RR (2007) Dynamic body weight and body composition changes in response to subordination stress. Physiol Behav 91:440-448.

Vallés A, Martí O, García A, Armario A (2000) Single exposure to stressors causes long-lasting, stress-dependent reduction of food intake in rats. Am J Physiol Regulatory Integrative Comp Physiol 279:R1138-R1144.

Watts AG (2001) Neuropeptides and the integration of motor responses to dehydration. Annu Rev Neurosci 24:357-384.

Watts AG, Salter DS, Neuner CM (2007) Neural network interactions and ingestive behavior control during anorexia. Physiol Behav 91:389-396.

Weingarten HP (1983) Conditioned cues elicit feeding in sated rats: a role for learning in meal initiation. Science 220:431-433.

Wilensky AE, Schafe GE, Kristensen MP, LeDoux JE (2006) Rethinking the fear circuit: the central nucleus of the amygdala is required for the acquisition, consolidation, and expression of Pavlovian fear conditioning. J Neurosci 26:12387-12396.

Woods SC (2005) Signals that influence food intake and body weight. Physiol Behav 86:709-716. 